\title{
Clinical analysis of mifepristone combined with transabdominal ultrasound-guided suction and curettage for different types of scar pregnancy
}

Hongmei Lian

Huazhong University of Science and Technology

dejun chen

Huazhong university of science and technology

Jingxia Zheng

Huazhong university of science and technology

Ling Zhang ( $\sim$ zhanglingxh@hust.edu.cn)

Huazhong university of Science and Technology

Research article

Keywords: cesarean scar pregnancy, mifepristone, suction curettage

Posted Date: February 12th, 2021

DOI: https://doi.org/10.21203/rs.3.rs-228066/v1

License: (c) (i) This work is licensed under a Creative Commons Attribution 4.0 International License.

Read Full License 


\section{Abstract \\ Background}

Cesarean scar pregnancy (CSP) is a life-threatening condition without consensus on treatment. The purpose of this study was to evaluate the safety, efficacy, and outcome of mifepristone combined with transabdominal ultrasound-guided suction and curettage (TUSC) in the treatment of CSP.

\section{Methods}

CSP patients who treated with mifepristone combined TUSC at Maternal and Child Hospital of Hubei Province from 2013 to 2015 was retrospectively analyzed. Patients were divides into three types based on the newest classification criteria in China. Clinical information and outcomes were analyzed.

\section{Results}

157 CSP patients were included, with 96 type I cases, 56 type II cases and 5 type III cases. No significant differences in age, gravity, parity, gestational age and initial $\beta$-human chorionic gonadotropin ( $\beta$-hCG) levels among the three types. All patients were successfully treated with mifepristone combined with TUSC. No one needed emergency blood transfusion or another treatment. After mifepristone combined with TUSC treatment, the percentage changes of the $\beta$-hCG levels were similar in all CSP types. The average of intraoperative blood loss in type I patients during suction curettage was lower than in type II and type III, and more Foley catheters were inserted into the uterus in type II and type III than type I patients to achieve hemostasis.

\section{Conclusion}

Mifepristone combined with TUSC appears to be a safety and efficacy treatment option to all types of CSP.

\section{Background}

Cesarean scar pregnancy(CSP), a special type of ectopic pregnancy, refers to the implantation of first trimester pregnancy within the scar or the niche of a previous cesarean section(CS)[1]. It occurs in 1:1800 to 1:2216 pregnancies, but increased significantly in recently decades due to the marked increasing rate of CS worldwide, the continuous development in diagnostic techniques, and the improved awareness of this condition[2]. CSP can often cause uterine rupture, uncontrolled hemorrhage, subsequent sterility, and even maternal death[3] and it is generally becoming accepted that CSP is a precursor of abnormally adherent placenta later in pregnancy[4], so early diagnosis and timely treatment are urged. 
Nowadays the optimal imaging modality for CSP diagnosis is transvaginal ultrasound (US)[1]. The Expert Consensus on Diagnosis and Treatment of Cesarean Scar Pregnancy published in the Chinese Journal of Obstetrics and Gynecology in 2016 classified CSP into three types (types I, II, and III) according to location and shape of the gestational substance, myometrium thickness in the incision region, and blood flow pattern in the incision region[5]. This classification appears to be helpful in the risk assessment and management options of CSP.

So far, there are many different treatment methods for CSP, but no optimal management strategy is suitable for both safety and effectiveness. The only consensus is that CSP should be terminated once confirmed[6]. Available treatments include medical therapy, ultrasound guided suction curettage, uterine artery embolization (UAE), laparoscopic excision, hysteroscopic resection and hysterectomy. Each has its advantages, limitations, and questionable drawbacks. Among them, transabdominal ultrasound-guided suction and curettage (TUSC) is a common method as it is a very simple and convenient. Some reports $[7,8]$ showed that TUSC is an effective method for successfully manage CSP but others reports indicated that it should not be considered as an optimal first line of therapy[9]. In recent years, more and more clinics chosen TUSC after UAE to intervene CSP patients [10]. However, UAE is associated with a number of complications, such as fever, pain, ectopic embolism, ovarian function injury and placenta accreta spectrum, which cause widespread concern[11]. Mifepristone has been reported as a safe and effective means of abortion, it can destroy the gestational villi, separate the villi from the uterine wall, reduce the blood flow around the gestational sac, which contributed to reduce intraoperative bleeding [12][13]. But no universal treatment guidelines for determining whether mifepristone combined with TUSC is effective in treating CSP and which type of CSP should be treated in this way. The purpose of this study was to evaluate the safety and effects of mifepristone combined with TUSC in CSP treatment.

\section{Methods}

\section{Study population}

A comparative observational study was conducted. We retrospectively analyzed our records from February 2013 to 2015 at Maternal and Child Hospital of Hubei Province, Tongji Medical college, Huazhong University of Science and Technology. 157 cases of CSP patients treated with mifepristone combined with TUSC were enrolled in this study. All patients gave written informed consent form before every procedure which clearly counseled the benefits, the curative effects, and the possible complications, including heavy vaginal bleeding and emergency hysterectomy. The study was approved by the ethics committee of Maternal and Child Hospital of Hubei Province. There were no conflicts of interest.

Patients were diagnosed with CSP relied on prior history of CS, increased $\beta$-human chorionic gonadotropin ( $\beta-h C G)$ level, and final postoperative pathological result. Patients were divided into three types according to the classification criteria of The Expert Opinion of Diagnosis and Treatment of Cesarean Scar Pregnancy. There were 96 type I cases (61\%), 56 type II cases (36\%) and 5 type III cases (3\%). The gestational age of patients was less than 12 weeks, without any coagulation disorders, 
trophoblastic disease and other disorders, and not receiving any treatment prior to admission. The vital signs of patients were stable.

\section{Administration of medicine}

In our study, patients were firstly treated with drugs. The method and dose of administration of medicine followed by the previous studies[14,15]. All patients took $50 \mathrm{mg}$ of mifepristone twice daily for 2 days at hospital: one at $10.00 \mathrm{~h}$ after breakfast and the other at $22.00 \mathrm{~h}$ before sleep, respectively. No patients suffered from uncontrollable vaginal bleeding during treatment.

\section{Transabdominal ultrasound-guided suction and curettage (TUSC)}

Suction and curettage under transabdominal ultrasound guidance was carried out as follows: Patients were in lithotomy position, transabdominal ultrasound was used to monitor the performance of the instruments. Firstly, adequately dilated the uterine cervical canal, secondly inserted suction curette along the posterior uterine wall to the uterine cavity, then aspirated the uterine fundus and posterior wall was with relatively higher suction pressure (about $400 \mathrm{mmHg}$ ), at last, placed the suction cannula at the gestational sac level to evacuate the conceptus content with lower suction pressure $(200-300 \mathrm{mmHg})$. If bleeding was detected, a Foley catheter $(16$ or $18 \mathrm{~F}$ ) was inserted into the uterine cavity and inflated with $10-20 \mathrm{ml}$ of saline placed in the region of the scar under sonographic guidance to compress the site of the bleeding. The Foley catheter was fixed to the leg with traction and uterine tamponade was continued for $24 \mathrm{~h}$.

\section{Follow-Up Observation}

Successful treatment was defined as disappearance of the CSP image on ultrasonography and with decreasing $\beta$-HCG levels, without complications, such as heavy bleeding or the need for hysterotomy and/or hysterectomy. Treatment failure was evidenced by the need for any additional nonsurgical or surgical treatment.

We recorded the clinical data of all these patients, including age, gravidity and parity, gestational days, results of ultrasound imaging examination (diameter of the gestational sac, thickness of the myometrial layer between the gestational sac and the bladder), the $\beta$-hCG level before surgery, the blood loss during surgery, and the $\beta$-hCG level 1 day after suction curettage postoperatively. All data were expressed as mean $\pm S D$. All patients were discharged from hospital 2 days after suction curettage. Following the approved protocol, the serum $\beta$-hCG level was monitored weekly until it returned to normal. The patients were requested to come back to our department for a color Doppler ultrasound examination 1 month after taking suction curettage.

\section{Statistical Methods}

Statistical analysis was performed by GraphPad Prism 5. The continuous data with a normal distribution were presented as means \pm standard deviations. One-way analysis of variance (ANOVA) was used for 
mean comparisons between groups. A P value of $<0.05$ was considered statistically significant.

\section{Results}

During the study period, our institution received a total of 157 CSP patients treated with mifepristone combined with TUSC. 96 patients were type I, 56 were type II and 5 were type III. The baseline parameters of subjects in the three types are provided in Table 1. There was no statistically significant difference in age, gestational age, gravidity, parity, abortion, previous cesarean sections, gestational age, preoperative size of CSP, and RI. As for Myometrial thicken, there were significant difference $(4.8 \pm 1.3 ; 2.4 \pm 0.5 ; 2.6 \pm$ $0.3, \mathrm{p} \otimes 0.0001$ ). In type II and type III patients, they have thinner Myometrial thicken (Table 1). This difference is inevitable because the Expert Opinion of Diagnosis and Treatment of Cesarean Scar Pregnancy is based primarily on the thickness of the myometrium.

Patients had similar initial serum $\beta$-hCG levels before mifepristone treatment in these three types of CSP (Table 1). When we examined $\beta$-hCG trends, we found that the mean decline rate of $\beta$-hCG after treatment was not significantly different among different types $(p=0.0811)$. In all types surveys before treatment and 1 days after curettage, the average $\beta$-hCG levels were similar.

During suction curettage, the blood loss was slight to moderate $(42.65 \pm 52.66$, from 10 to $300 \mathrm{ml})$. All patients had successful resolution of the CSP without complications. No one required emergency blood transfusion or UAE. Although treatments demonstrated high success rates but there was significantly statistical difference among the three types. The average of intraoperative blood loss in type I patients during suction curettage was lower than in type II and type III ( $28.6 \pm 37.9$ vs $64.2 \pm 65.7$ vs $71.0 \pm$ $50.0 \mathrm{~mL}, \mathrm{P} \otimes 0.0001)$. what's more, Foley catheters were inserted into the uterus more common in type II and type III than type I patients to achieve hemostasis (Table 2).

\section{Discussion}

CSP, first proposed in 1978 by Larsen and Solomon[16] is an extremely rare form of ectopic pregnancy. Although nowadays there has been an increasing awareness of this condition, the incidence of CSP are underreported[11] and there were no clear etiology and no consensus guideline for the management of CSP. Considering the life-threatening risk (uterine rupture and uncontrolled catastrophic hemorrhage) of patients with CSP, early diagnosis and better management strategies were urgently needed[17].

CSP is classified in different ways at different times and in different regions. In 2000 defined CSP into two types[18]. Gestational sac in CSP-1 patients locates in the existing scar and grows toward the uterine cavity, while in CSP-2 it implants deeply into the scar defect and grows towards the myometrium and uterine serosal layer. According to the expert consensus in China in 2016[5], CSP is classified as three types (types I, II, and III) based on the relationship between the gestational sac and uterine incision scar by ultrasound. As for type I patients, the myometrium thickness of the scar in the anterior uterine wall is $₫ 3 \mathrm{~mm}$ while $\varangle 3 \mathrm{~mm}$ for type II and III. In type II, partial gestational sac implanted in the muscle layer of 
the uterine scar, in type III, the whole gestational sac implanted and grows toward the bladder, and it may always cause serious consequences. Because of the difference in sac invasion degree and clinical consequences, the three types of CSP may differ in treatment choice and prognosis.

An appropriate treatment should be both safe and effective, termination of pregnancy, minimization of hemorrhage while preserving conservation of fertility. Is there an appropriate treatment for all three types of CSP? Many treatments have been recommended, including conservative medical and radical surgery, which can be used alone or in combination. Almost all methods have successful cases, none is clearly considered to be the Most appropriate[19]. Reports have showed that ultrasound guided suction curettage was a reliable option for CSP treatment[7]. Due to the insertion of villus into the muscular layer and lacking of effective contraction, curettage directly is dangerous as it could cause hemorrhaging and even shock. So, in the present study, we introduced mifepristone to destroy the gestational villi and separate the villi from the uterine wall to reduce bleeding. To our surprise, mifepristone combined with TUSC was safe and effective in treating all types of CSP, although in types II and III intraoperative blood loss were mild larger and Foley catheters uses were more common. In the present study, mifepristone combined with TUSC is our first choice of treatment for patients with hemodynamic stability. we showed that age, gestational age, gravidity, parity, abortion, previous cesarean sections, gestational age, preoperative size of CSP, and RI are no difference in the three types of CSP, but myometrial thicken were significant difference. According to the previous report [20], treatment was defined as satisfactory when the decline in hCG levels was $>15 \%$ in a week, based on this all patients achieved satisfactory results regardless of CSP type.

There were some limits in our study. First, the study is in a retrospective manner and includes patients in a single center, which could not exclude a selection bias. Second, we didn't monitor vaginal bleeding time, the first menstrual period, duration of abnormal blood $\beta-h C G$, and fertility outcome of patients after the treatment.

\section{Conclusion}

Based on our study we can offer management strategies for patients with CSP as follows: mifepristone combined with TUSC can concern as the first-line approach for different types of CSP patients that are hemodynamically stable. But large-scale randomized prospective studies without bias for the purpose of identifying reliable and fore feasible first line treatments are essential.

\section{Declarations}

Ethics approval and consent to participateIThe study was approved by the Ethics Committee of Maternal and Child Hospital of Hubei Province, Tongji Medical college, Huazhong University of Science and Technology

Consent for publicationロNot Applicable. 
Availability of data and materials $\square$ The datasets included in the current study are available from the corresponding author on reasonable request.

Competing interests: The authors declare no conflicts of interest.

Authors' contributions: Zhang Ling drafted and revised the manuscript, and analysed and interpreted data for the work. Hongmei Lian designed the work, wrote and contributed to the manuscript. Dejun Chen was responsible for conceiving the work and performing all the procedures. Jingxia Zheng collected and analysed the data. All authors have read and approved the manuscript.

Funding: This project was supported by Foundation of Maternal and Child Hospital of Hubei Province

Informed consent: Informed consent was obtained from all individual participants included in this study.

Acknowledgements: Not Applicable.

\section{References}

1. Society for Maternal-Fetal Medicine . Electronic address pso, Miller R, Timor-Tritsch IE, Bannerman CG (2020) Society for Maternal-Fetal Medicine (SMFM) Consult Series \#49: Cesarean scar pregnancy. Am J Obstet Gynecol. doi:10.1016/j.ajog.2020.01.030

2. Jurkovic D, Knez J, Appiah A, Farahani L, Mavrelos D, Ross JA (2016) Surgical treatment of Cesarean scar ectopic pregnancy: efficacy and safety of ultrasound-guided suction curettage. Ultrasound Obstet Gynecol 47 (4):511-517. doi:10.1002/uog.15857

3. Wang G, Liu X, Bi F, Yin L, Sa R, Wang D, Yang Q (2014) Evaluation of the efficacy of laparoscopic resection for the management of exogenous cesarean scar pregnancy. Fertil Steril 101 (5):15011507. doi:10.1016/j.fertnstert.2014.01.045

4. Harb HM, Knight M, Bottomley C, Overton C, Tobias A, Gallos ID, Shehmar M, Farquharson R, Horne A, Latthe P, Edi-Osagie E, MacLean M, Marston E, Zamora J, Dawood F, Small R, Ross J, Bourne T, Coomarasamy A, Jurkovic D (2018) Caesarean scar pregnancy in the UK: a national cohort study. BJOG 125 (13):1663-1670. doi:10.1111/1471-0528.15255

5. Family Planning Subgroup CSoO, Gynocology CMA (2016) [Expert opinion of diagnosis and treatment of cesarean scar pregnancy (2016)]. Zhonghua Fu Chan Ke Za Zhi 51 (8):568-572. doi:10.3760/cma.j.issn.0529-567X.2016.08.003

6. Guo J, Yu J, Zhang Q, Song X (2018) Clinical Efficacy and Safety of Uterine Artery Embolization (UAE) versus Laparoscopic Cesarean Scar Pregnancy Debridement Surgery (LCSPDS) in Treatment of Cesarean Scar Pregnancy. Med Sci Monit 24:4659-4666. doi:10.12659/MSM.907404

7. Ozcan HC, Ugur MG, Balat O, Sucu S, Mustafa A, Bayramoglu Tepe N, Ugur BK (2018) Is ultrasoundguided suction curettage a reliable option for treatment of cesarean scar pregnancy? A cross- 
sectional retrospective study. J Matern Fetal Neonatal Med 31 (22):2953-2958.

doi:10.1080/14767058.2017.1359827

8. Ozdamar O, Doger E, Arlier S, Cakiroglu Y, Ergin RN, Kopuk SY, Caliskan E (2016) Exogenous cesarean scar pregnancies managed by suction curettage alone or in combination with other therapeutic procedures: A series of 33 cases and analysis of complication profile. J Obstet Gynaecol Res 42 (8):927-935. doi:10.1111/jog.13017

9. Wang S, Li Y, Ma X (2018) Lower uterine segment thickness in assessing whether cesarean scar pregnancy patients could be treated with suction curettage. J Matern Fetal Neonatal Med:1-6. doi:10.1080/14767058.2018.1531118

10. Ou J, Peng P, Li C, Teng L, Liu X (2020) Assessment of the necessity of uterine artery embolization during suction and curettage for caesarean scar pregnancy: a prospective cohort study. BMC Pregnancy and Childbirth 20 (1). doi:10.1186/s12884-020-03062-z

11. Timor-Tritsch IE, Monteagudo A (2012) Unforeseen consequences of the increasing rate of cesarean deliveries: early placenta accreta and cesarean scar pregnancy. A review. Am J Obstet Gynecol 207 (1):14-29. doi:10.1016/j.ajog.2012.03.007

12. Yang Q, Piao S, Wang G, Wang Y, Liu C (2009) Hysteroscopic surgery of ectopic pregnancy in the cesarean section scar. J Minim Invasive Gynecol 16 (4):432-436. doi:10.1016/j.jmig.2009.03.015

13. Luo X, Shu S-r, Wang Z-x, Yao Y-h (2015) Cesarean scar pregnancy treated by curettage and aspiration guided by laparoscopy. Therapeutics and Clinical Risk Management:1139. doi:10.2147/tcrm.s86083

14. Xu J, Chen H, Ma T, Wu X (2001) Termination of early pregnancy in the scarred uterus with mifepristone and misoprostol. Int J Gynaecol Obstet 72 (3):245-251. doi:10.1016/s00207292(00)00341-6

15. Wang G, Li D, Manconi F, Dong B, Zhang Y, Sun B (2010) Timing and indication for curettage after medical abortion in early pregnant women with prior uterine incision. Contraception 81 (1):62-66. doi:10.1016/j.contraception.2009.09.013

16. Larsen JV, Solomon MH (1978) Pregnancy in a uterine scar sacculus--an unusual cause of postabortal haemorrhage. A case report. S Afr Med J 53 (4):142-143

17. Gibbons L, Belizan JM, Lauer JA, Betran AP, Merialdi M, Althabe F (2012) Inequities in the use of cesarean section deliveries in the world. Am J Obstet Gynecol 206 (4):331 e331-319. doi:10.1016/j.ajog.2012.02.026

18. Vial Y, Petignat P, Hohlfeld P (2000) Pregnancy in a cesarean scar. Ultrasound Obstet Gynecol 16 (6):592-593. doi:10.1046/j.1469-0705.2000.00300-2.x

19. Birch Petersen K, Hoffmann E, Rifbjerg Larsen C, Svarre Nielsen H (2016) Cesarean scar pregnancy: a systematic review of treatment studies. Fertil Steril 105 (4):958-967. doi:10.1016/j.fertnstert.2015.12.130

20. Sadeghi H, Rutherford T, Rackow BW, Campbell KH, Duzyj CM, Guess MK, Kodaman PH, Norwitz ER (2010) Cesarean scar ectopic pregnancy: case series and review of the literature. Am J Perinatol 27 
(2):111-120. doi:10.1055/s-0029-1224874

\section{Tables}

Table 1. Patient clinical data in the three types of CSP

\begin{tabular}{lcccc}
\hline & Type I & Type II & Type III & P value \\
\hline Number of cases & 96 & 56 & 5 & - \\
Age (Years) & $31.9 \pm 4.7$ & $31.2 \pm 4.2$ & $32.6 \pm 5.0$ & 0.5749 \\
Gestational age (Days) & $49.3 \pm 9.5$ & $48.8 \pm 8.7$ & $44.8 \pm 5.8$ & 0.5526 \\
Gravidity & $3.9 \pm 1.4$ & $4.1 \pm 1.5$ & $3.4 \pm 1.1$ & 0.4039 \\
Previous casarean section & $1.2 \pm 0.4$ & $1.3 \pm 0.5$ & $1.2 \pm 0.4$ & 0.4801 \\
Time interval (years) & $4.0 \pm 2.7$ & $4.3 \pm 3.4$ & $5.0 \pm 3.3$ & 0.6591 \\
Myometrial thicken(cm) & $4.8 \pm 1.3$ & $2.4 \pm 0.5$ & $2.6 \pm 0.3$ & $<0.0001$ \\
Mass size (cm) & $1.7 \pm 0.9$ & $1.9 \pm 0.9$ & $1.4 \pm 0.3$ & 0.2381 \\
RI & $0.4 \pm 0.1$ & $0.4 \pm 0.1$ & $0.5 \pm 0.1$ & 0.0094 \\
$\beta$-hCG (mlU/ml) & $43090 \pm$ & $53264 \pm$ & $34792 \pm$ & 0.5451 \\
& 66055 & 49560 & 22102 & \\
\hline
\end{tabular}

Table 2. Comparison of therapeutic effects in the three types of CSP

\begin{tabular}{lcccc}
\hline & Type I & Type II & Type III & P value \\
\hline Surgical hemorrhage $(\mathrm{ml})$ & $28.6 \pm 37.9$ & $64.2 \pm 65.7$ & $71.0 \pm 50.0$ & $<0.0001$ \\
Serum $\beta$-hCG decline rate $(\%)$ & $68.6 \pm 14.0$ & $72.1 \pm 8.5$ & $61.5 \pm 14.6$ & 0.0811 \\
Foley catheter $\mathrm{n}(\%)$ & $4(4 \%)$ & $16(29 \%)$ & $2(40 \%)$ & - \\
\hline
\end{tabular}

